\title{
Unravelling the Negative Impact of Pain Problems on Recovering from Psychological Trauma
}

\author{
Carol Valinejad, Steven Lang \\ Trauma Services, Salus Psychological Services Ltd, London, England
}

Email address:

drvalinejad $a$ saluspsychological.com (C. Valinejad), francisslang@ hotmail.co.uk (S. Lang)

To cite this article:

Carol Valinejad, Steven Lang. Unravelling the Negative Impact of Pain Problems on Recovering from Psychological Trauma. Clinical Medicine Research. Vol. 8, No. 1, 2019, pp. 27-31. doi: 10.11648/j.cmr.20190801.15

Received: October 16, 2018; Accepted: April 9, 2019; Published: May 9, 2019

\begin{abstract}
Background: Pain problems can act as a barrier to individuals recovering from psychological trauma following receiving appropriate psychological therapy. Therefore, it would be prudent of therapists to treat pain problems in clients prior to embarking on trauma informed therapy to increase the chances of therapy being more clinically and cost effective. Objective: This article aims to provide practice-based evidence to demonstrate how a client's pain problems were affecting her recovery from severe psychological trauma following a serious accident, and how psychological pain management work prior to her starting trauma informed therapy assisted her to overcome the barrier of pain to her recovery. Method: The client was offered psychological pain management intervention based on the gate theory of pain prior to embarking on trauma focussed cognitive behavioural therapy to treat her post trauma symptoms following a serious accident. The gate theory argues that psychological factors have potential to open a gate to the pain system causing an increase in pain perception. According to this model, once these factors are addressed it will lead to improvement in pain perception. Result: Guided imagery techniques were utilised to assist the client to resolve emotional factors in connection with her pain which resulted in further recovery of her pain perception. Following recovery in this the client was able to experience increase emotional capacity as well as mobility to receive trauma focussed cognitive behavioural therapy to reduce her psychological trauma symptoms. Conclusion: This case study was able to demonstrate that treating clients pain problems prior to treating psychological trauma has potential to be clinically and cost effective. It is recommended that further research be done in this area.
\end{abstract}

Keywords: Trauma-Focussed, Pain, Recovery

\section{Introduction}

Salus Psychological Services is specialist in delivering trauma informed psychological treatments and is always exploring ways to provide efficient psychotherapy to resolve people's distress as soon as possible [1]. Many of its referrals are of individuals with a history of complex presentations, who may have sought help from a multiplicity of service providers' prior to being referred. Indeed, the fact that they seek further help from Salus Psychological Service is because in spite of receiving numerous assessments and interventions previously, they have not received a satisfactory resolution to their problems. Indeed, by the time they start treatment with the service many clients have reached a place of hopelessness believing that they are unable to be helped.

Trauma can present itself in many guises, for some, the trauma is not just a result of a traumatic event, but it could also be further compounded by pain related injuries and the subsequent impact of both psychological and physical consequences on the individual's life [2] Research shows that individuals with chronic pain are reported to have associated mood related problems [3]. In fact, relative to patients without persistent pain, pain sufferers were more likely to have unfavourable health perceptions, to experience significant activity limitations and more likely to have a diagnosis of an anxiety or depressive disorder $[4,5]$. As such, it is reasonable to assume that individuals suffering from persistent pain are more likely to struggle to recover from psychological trauma due to the considerable strain that their pain puts on their well-being. Pain related problems present considerable barriers to individuals benefitting from treatment that has potential to resolve psychological trauma and it is the reason pain is often used as an external factor on 
treatment reports to explain why a certain form of therapy has not been of benefit. It therefore follows that in order to increase a pain suffers receptiveness to treatment that will resolve their psychological trauma, psychological pain management work is required prior to this. One theory of pain which has underpinned psychological pain management approaches in recent years is the Gate Theory of Pain [6]. According to this theory there are gates in nerve junctions, spinal cord and pain centres in the brain. These gates open and let pain messages through the pain system, so that can be experienced. They can also close to stop messages going through the system, so that the pain is reduced or stopped. Sonner summarizes this model by stating,

"Pain is best conceptualized as a perceptual, rather than a sensory, phenomenon. As a purely sensory event, pain is a response to tissue damage generated by a stimulus to a nociceptor. Patients, however, have vastly different responses to very similar pain-generating stimuli, suggesting that the patients' perception of pain cannot be caused solely by the intensity of the initial stimulus. Viewing pain in a perceptual framework allows a much greater recognition of the attentional, cognitive, affective, and social components to the pain experience. Psychotherapeutic approaches using perceptual frameworks can successfully treat some forms of pain." [7].

It is upon the Gate Theory of Pain that a psychological pain management approach is described prior to embarking on trauma informed therapy to assist a client to recover from her post trauma symptoms following a serious accident.

\section{Psychological Intervention}

\subsection{Case Study}

A case study of a 51-year-old woman from an African Caribbean background will be described. In order to preserve her anonymity, she will be referred to as Sally. She was informed about the service evaluation of the study and provided consent for her story to be used for the purposes of the article .Sally suffered considerable injuries whilst travelling to work on a tram in London, England; in the early hours of the morning some two years ago the tram Sally was travelling on, derailed and overturned due to excessive speeds and driver error. As the tram hit the ground, the windows shattered, and this led to multiple passengers being thrown out of the cab. The accident led to the death of seven passengers and the serious injury of another 19, of which, Sally was one. As a result, Sally developed considerable psychological and physical trauma, attributed to the events she experienced and witnessed. In addition, on account of the extent of her physical injuries she had to give up her job as a cleaner in a well-known hotel.

Sally had initially been provided with 12 sessions of trauma focussed Cognitive Behavioural Therapy from another provider, but upon the completion of this, was still experiencing some post-trauma reactions; she was subsequently referred to me to resolve any residual symptoms. Since starting therapy with me she has been seen for a total of 30 sessions. It is acknowledged that this is a particularly large number of sessions to be offering efficient psychotherapy, however it was justified for the following reasons: One, as she was initially funded for psychological treatment to address her psychological symptoms this was the initial target of her treatment for a number of weeks until her difficulties were re-formulated as being a primary pain related problem which was acting as a barrier to her making use of trauma therapy in this respect. Two, in addition to the trauma which she suffered due to personal injury, she also suffered the negative impact of multiple losses since her accident, namely loss of her functionality and her subsequently her job which was in stark contrast to the busy and active life which she had prior to the accident. The treatment therefore has targeted three areas, namely her pain related problems, her post trauma and situational anxiety symptoms and her "grief reaction" to her losses.

Her presenting psychological difficulties at the beginning of this episode of therapy and subsequent to her receiving previous psychological treatment were: situational anxiety in respect to leaving her house and car travel, an exaggerated startle reaction to loud noises and fear of the dark. As a result of her exaggerated startle reaction and fear of the dark she had subsequently avoided going to the cinema on account of the loud sounds, which she previously enjoyed attending regularly. In addition, her fear of the dark had led her into needing to leave the television on throughout the night, as to avoid sleeping in the dark.

\subsection{Reformulating the Focus of Therapy}

Intially, her treatment aimed to address her post-trauma symptoms and situational anxiety, much like the previous therapist. However, following this, it soon became apparent that due to the large impact her pain problems were having on her functioning, it was difficult to progress through this line of enquiry. Sally's pain related problems were negatively impacting her in the following ways: she was unable to attend her domestic tasks such as tidy her house and selfcare. Sally required a carer to come into her home to wash her and help her with daily household tasks. As a result of the pain, Sally would spend most of her day sitting down, watching TV, and would only move around the house for essential reasons. The only physical intervention that Sally was offered at the time was pain killers. She has since been offered physiotherapy and hydrotherapy, but this has targeted her mobility issues rather than her pain.

Sally reported experiencing pain all over her body. See fig 1 to illustrate this. She described the pain in different parts of her body in different ways as she seemed to have a different relationship with each one. For example, with regards to the pain in her neck, she was particularly rejecting of it, as it was an area where she had sustained a scar in the tram accident which she avoided looking at. In fact, she avoided looking at it, at all costs, due to the extent that she would even wear a scarf in public around it. This was despite her scar not looking very apparent to the naked eye. In contrast, even 
though Sally experienced pain in her hands and feet, she was more nurturing of these areas of her body. For example, she regularly received a manicure and pedicure. Considering the extent to which Sally's pain was affecting her day to day well-being, the decision was taken to target this during therapy.

\section{Psychological Pain Management}

\subsection{The Gate Theory of Pain}

The Gate Theory Model of pain was utilised to assist Sally to manage her pain [6]. Sally was diagnosed with Chronic Pain and according to gate theory there are no treatments to shut the gate and keep it shut all the time. However, there are ways to close the gate as much as possible so that fewer pain messages pass through the pain system. Initial work therefore assisted Sally to identify what activities she was doing which served to open her gate and thus increased her pain perception. This model suggests that activities such as staying in one position for too long, poor pacing of activities, experiencing emotional issues such as worry or stress, getting angry, getting frustrated and not enough sleep can serve to open the gate and thus cause an increase in pain perception. Whereas in contrast a reduction in these activities can cause a decrease in pain perception. These will be reviewed in turn and analysed in respect to their relevance to Sally's experience of pain, and what interventions were utilised to assist Sally to close her pain gate.

\subsection{Mapping Gate Theory onto Personal Experience}

\section{Emotional Issues}

Sally had suffered marked negative impact to her mood due to the restrictions which were imposed upon her due to her physical injuries. She discussed how prior to the accident she was an active independent person, working as a full time cleaner and always out and about. However, since her accident she had lost her job, unable to attend to most of her self-care and domestic activities on account of her pain requiring a carer to assist her with this. In addition, she had stopped many of her former past times on account of her marked reduced mobility on account of her pain as well as her post trauma symptoms. This predicament often left Sally feeling "stuck" whenever she felt that she was powerless to change things. She subsequently would become depressed about her predicament. Any intervention therefore to assist Sally to feel less "stuck" was of assistance to her. Part of this intervention helped to reduce her levels of pain which in turn helped to increase her level of mobility. This is described in more detail in the section below.

Increasing her mobility

Sally was asked to monitor her pain on a weekly basis in order to get some idea of the levels of pain she was experiencing. She identified pain in her neck, shoulders, chest, spine, hips fingers which she rated as either in the severe or moderate range (see fig 1 pre-treatment). Each area of her pain was worked on in turn, using guided imagery to conceptualise and directly confront her pain. Guided imagery involves the generation of specific mental images to evoke a state of relaxation or physiologic change [9], $\{10\},[11]$, such as, the imagining of pain from a headache being collected in a balloon and slowly drifting away. Although, it is difficult for some people to accept that pain can have a psychological as well as physical root, randomised-controlled studies have found that guided imagery has significantly reduced various types of pain [8]. During one session with Sally, she reported that she was suffering with pain on her spine, which almost felt like someone was "playing it like an instrument". I used guided imagery to propose that Sally imagines the musician pulling the instrument away from her spine and placing it down. The combination of this with progressive muscle relaxation led to Sally reporting a reduction in her pain, solely in the short time of our session. See fig 1 to illustrate changes in Sally's pain perception from pre-treatment to the time the psychological pain management intervention was complete.

Over the course of our sessions, Sally continued to progress, and we continued to experiment with imagery work. She started to notice small improvements to her physical symptoms; her finger and toe pain were much reduced, and she was beginning to be able to move her arms further than she was previously able. Sally acknowledges that due to the extent of her injuries, she is unlikely to ever have full movement or a complete lack of pain again, however, she has been able to improve her situation and is beginning to see a future in which she is able to enjoy her life again. In order to keep herself mobile, Sally began to take more responsibility to engage in activities to keep her active. She has started to walk up and down her garden path at least once per day. She accepts to go on social outings with her sister more often. Prior to pain management work, she was unable to cook for herself due to the pain she experienced with lifting pots and pans, cutting food and bending down, but has gradually started to cook small and simple meals where not too much physical demands are placed upon her. She had also been able to travel on holiday for one week for the first time with members of her extended family and although it was a challenge to keep up with all of the activities leaving her with additional pain she did it none the less.

This work helped to improve her mood and she began to feel less stuck.

Sleep Disturbance

Since her accident, Sally had suffered sleep disturbance. Whereas prior to the accident she used to sleep through out the night, following her tram accident she was only sleeping for up to 4 hours per night. One of the reasons Sally gave to account for her sleep disturbance was due to her neck pain. Sally had sustained a neck injury following the accident which required an operation. Although it was not noticeable, Sally expressed particular sensitivity to the area on her neck where she developed a scar. She avoided looking at her scar and even touching it. She seemed to reject this area of her body and in her attempts to avoid being reminded of it she wore a scarf around it whenever she went out in public and 
would avoid touching this area of her neck whenever she had a shower. Sally was able to respond to the suggestion that she needed to learn to love her neck again if she wanted it to respond in a more functional way. She engaged in exposure therapy coupled with relaxation intervention to assist her to become habituated to the distress she experienced whenever she caught sight of the scar on her neck. Over time, Sally's distress associated with her neck decreased to the point she noticed that on occasions when she went out she had forgotten to wear her scarf, thus exposing her neck unawares. In addition, she started to allow her carer to wash the area around her neck whereas previously she was ashamed to expose this area to her carer. Interestingly, over time Sally reported that her sleep had improved. She is now having up to 5 hours after this intervention whereas prior to it she was only having 4 hours per night. Interestingly, it would appear that once Sally accepted this injured area of her body, she seemed to note improved pain perception. [13] found that by encouraging individual's to distance themselves from their private events, acceptance methods may help reduce the use of emotional reasons to explain behaviour and hence shift concern from moderating thoughts and feelings to experiencing the consequences of one's action.

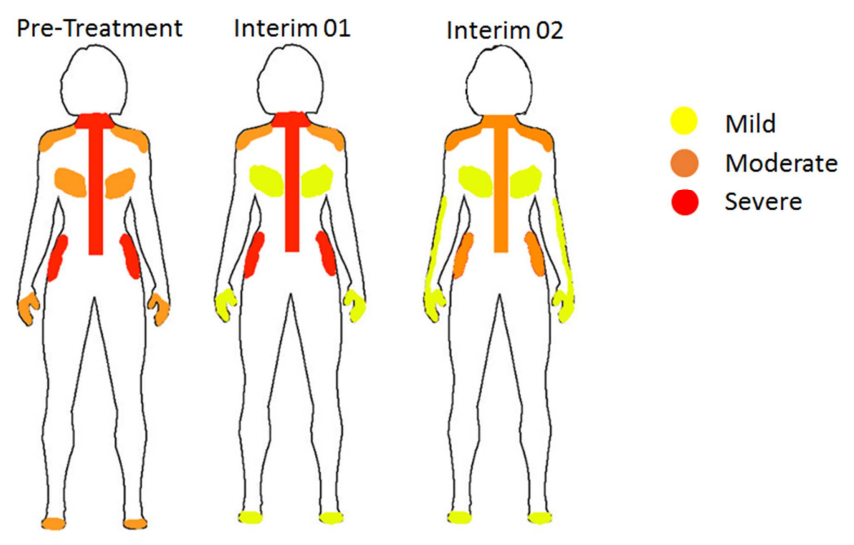

Figure 1. Sally's pain maps.

\section{Evaluation}

Sally had used pain-killers daily since the tram accident and required them to complete daily activities. Since Sally has begun psychological pain management work and she has noticed improvements in her psychological as well as physical well-being to the extent that can now achieve more, there has been a corresponding reduction in her use of pain killers. She now uses her pain killers strategically rather than out of compulsion. For example, if she has a number of activities planned, she will use her pain killers as a prevention of experiencing high levels of pain. Sally has found that it is only since the introduction of psychological pain management that she is beginning to see improvements in her condition and in her ability to cope with her pain.

Once her physical symptoms had improved, Sally became more amenable to addressing her psychological symptoms. For example, given that she experienced improved mobility she was now able to physically confront her fears. With the use of Cognitive Behavioural Therapy, she experienced recovery from her exaggerated startle reaction, and fear of the dark. This work had a positive impact on her life as she has been able to visit the theatre and cinema which she was previously unable to do since the accident on account of her fear of the dark and intolerance of loud noises. She even reported that she had been able to enjoy the display of fireworks last new year, whereas previously she had suffered much distress when she heard them. Ongoing work will assist her to manage her anxiety with regards to travelling past the scene of the accident in a taxi.

Sally has been administered with the Clinical Outcomes in Routine Evaluation outcome measure [14]. Her score at beginning of treatment was 1.76 and by the time she completed pain related work it reduced to 1.14 which suggests a reduction in psychological distress from the clinically significant to the non-clinically significant range.

Given the considerable impact that this accident has had upon her life, namely the losses she has encountered such as her job and her adjustment to a more limited lifestyle. Sally continues to be seen for ongoing therapy to help her come to terms with what has happened to her. The plan is to support her eventual discharge by allowing her more infrequent sessions to support independent self -management.

\section{Conclusion}

The aim of this article was to demonstrate using a case study approach how by using psychological pain management approaches to reduce a client's pain, can increase an individual's capacity to receive trauma informed therapy to resolve their psychological trauma. From this case study, improvements in Sally's pain related problems were noted and although all of her pain not ceased, her activity levels have improved from the point of being completely house bound to socialising frequently with family members and travelling abroad with family for the first time. Her increased sociability following treatment paved the way for her to commence trauma informed treatment to resolve her post trauma symptoms following a serious accident. This demonstrates that clients where pain related problems act as a barrier to them recovering from their post trauma symptoms following an accident can be assisted to manage their pain related problems prior to embarking on trauma informed therapy.

This case study confirms that clients who have suffered both psychological trauma concurrently with pain problems would benefit by receiving psychological pain management work prior to embarking on trauma informed treatment to relieve their post trauma symptoms. This is likely to lead to a more efficient delivery of therapy sessions. Sally had already received a considerable number of CBT sessions for her psychological trauma symptoms with limited success prior to commencing psychological pain management work. If she had commenced psychological pain management intervention prior to embarking on trauma informed therapy, 
it is possible that she would have recovered from her post trauma symptoms sooner.

Sally's use of pain killers was also noted to have reduced following this intervention. Whilst previously she relied on pain killers to function, she progressed to functioning without them and will only use pain killers occasionally when her pain levels are particularly high. This outcome could have economic implications. Current statistics reveal that the NHS spends almost $£ 1,000,000$ a year on solely prescribing painkillers, which is only expected to rise [15]. This put's a vast strain on the NHS service and should spur researchers and clinicians into experimenting and finding more efficient and cost-effective ways of managing pain.

This study constitutes practice-based evidence using one case study, so the findings may not be generalisable. However, given the clinical and potential cost benefit of treating pain problems prior to treating psychological trauma in this case, it is recommended that further research is carried out to establish the clinical and cost benefit in treating pain problems prior to treating psychological trauma.

\section{References}

[1] Valinejad, C (2005) Delivery efficient psychotherapy, Clinical Psychology Forum, 271, 33-35.

[2] Vranceanu, A. M., Bachoura, A., Weening, A., Vrahas, M., Smith, R. M., \& Ring, D. (2014). Psychological factors predict disability and pain intensity after skeletal trauma. $J B J S$, 96(3), e20.

[3] Feldman, S. I., Downey, G., \& Schaffer-Neitz, R. (1999). Pain, negative mood, and perceived support in chronic pain patients: A daily diary study of people with reflex sympathetic dystrophy syndrome. Journal of Consulting and Clinical psychology, 67(5), 776.

[4] Gureje, O., Von Korff, M., Simon, G. E., \& Gater, R. (1998). Persistent pain and well-being: a World Health Organization study in primary care. Jama, 280(2), 147-151.
[5] Fishbain, D. A., Cutler, R., Rosomoff, H. L., \& Rosomoff, R. S. (1997). Chronic pain-associated depression: antecedent or consequence of chronic pain? A review. The Clinical journal of pain, 13(2), 116-137.

[6] Melzack, R, Wall, P. D (1965) Pain Mechanisms: A New Theory. Science, 971-976.

[7] Songer, D. (2010). Psychotherapeutic approaches in the treatment of pain. Psychiatry (Edgmont), 2(5), 19.

[8] Ackerman, C; Turkoski, B (2000) Using Guided Imagery to Reduce Pain and Anxiety, Home Healthcare Nurse, Vol18 (8) - 524-530.

[9] Menzies, V; Taylor, A. G; Bourguignon, C (2006). Effects of Guided Imagery on Outcomes of Pain, Functional Status, and Self-Efficacy in Persons Diagnosed with Fibromyalgia. The Journal of Alternative and Complementary Medicine, Vol. 12, No. 1

[10] Hayes, S. C; Bissett, R. T; Korn, Z; Zettle, R. T; Rosenfarb, I. $S$ (1999). The Impact of Acceptance Verses Control Rationales on Pain Tolerance, The Psychological Record, Vol, 149, Issues 1, pp 33-47.

[11] Cole, F; Macdonald, H; C Carus, H Howden-Leach (2005) Overcoming Chronic Pain. Robinson. UK.

[12] Bonny, H. L. (2002). Music consciousness: The evolution of guided imagery and music. Barcelona Publishers.

[13] Giacobbi Jr, P. R., Stabler, M. E., Stewart, J., Jaeschke, A. M., Siebert, J. L., \& Kelley, G. A. (2015). Guided imagery for arthritis and other rheumatic diseases: a systematic review of randomized controlled trials. Pain Management Nursing, 16(5), 792-803.

[14] Evans, John Mellor-Clark, Frank Margison, Michael Barkham, Kerry Audin, Janice Connell, Graeme McGrath, C. (2000). CORE: clinical outcomes in routine evaluation. Journal of Mental Health, 9(3), 247-255.

[15] Ewbank, L., Omojomolo, D., Sullivan, K., \& McKenna, H. (2018). The rising cost of medicines to the NHS. The Kings Fund. 